\title{
Desarrollo mecánico y experimental de un implante discal cervical flexible
}

\author{
Mechanical and experimental development of a flexible cervical disc implant
}

\author{
Carlos Alvarez ${ }^{1,2, a}$, Paul Lean ${ }^{3, \mathrm{~b}}$, Rodolfo Rodríguez ${ }^{1,2, \mathrm{c}}$, Claudio Pacussich $^{1,2, \mathrm{~d}}$, Ainsworth Noriega ${ }^{3, \mathrm{e}}$, \\ Erick Navarro ${ }^{3, f}$ \\ ${ }^{1}$ Departamento de Neurocirugía, Clínica Álvarez. Lima, Perú. \\ ${ }^{2}$ Instituto del Cerebro y la Columna Vertebral. Lima, Perú. \\ ${ }^{3}$ Pontificia Universidad Católica del Perú. Lima, Perú. \\ ${ }^{a}$ Neurocirujano, ORCID: http://orcid.org/0000-0002-0043-9801 \\ ${ }^{b}$ Ingeniero mecánico, ORCID: https://orcid.org/0000-0001-9411-6984 cNeurocirujano, ORCID: https://orcid.org/0000-0001-6361-3451 \\ ${ }^{d}$ Neurocirujano, ORCID: http://orcid. org/0000-0003-0898-6751 \\ ${ }^{e}$ Ingeniero mecánico, ORCID: https://orcid.org/0000-0002-0317-321X \\ ${ }^{\dagger}$ Ingeniero mecánico, ORCID: http://orcid.org/0000-0002-0043-9801
}

An Fac med. 2019;80(3):305-11 / DOI: https://10.15381/anales.803.16105

\section{Correspondencia: \\ Carlos Eduardo Álvarez Peña c.alvarez@iccvperu.com}

Recibido: 13 de mayo 2019

Aceptado: 31 de agosto 2019

Publicación en línea: 30 de setiembre 2019

Conflictos de interés: Los autores declaran no tener conflictos de interés.

Fuente de financiamiento: Innóvate Perú, Ministerio de la Producción del Perú. Convenio No 724 - Innóvate Perú - Proyecto de Innovación de Empresas, 2017.

Citar como: Alvarez C, Lean P, Rodríguez R, Pacussich C, Noriega A, Navarro E. Desarrollo mecánico y experimental de un implante discal cervical flexible. An Fac med. 2019;80(3):305-11. DOI: https://10.15381/anales.803.16105

\section{Resumen}

Introducción: La artroplastía cervical es una cirugia que implica el uso de implantes discales con la capacidad de conservar el movimiento del segmento. Constituye un reto para paises como el nuestro desarrollar alternativas a los altos costos de los mencionados implantes, con diseños patentado, elaborado viables de manufacturar, con biocompatibilidad, seguridad, y que simule lo máximo posible los movimientos cervicales fisiológicos. Objetivo: Desarrollar la geometría y mecánica de un diseño discal patentado, elaborado, sea por manufactura aditiva de titanio ELI, así como verificar su compatibilidad con la fisiología de los movimientos cervicales. Métodos: El diseño del estudio tuvo tres etapas iterativas: la primera, el desarrollo de la geometría del implante y el estudio de elementos finitos utilizando como referencia normas internacionales; en segundo lugar, se desarrollaron estudios de metalografia, caracterización del material incluyendo pruebas de fatiga del implante; finalmente, se utilizó un modelo animal cadavérico para confirmar la compatibilidad del implante con los movimientos cervicales. Resultados: Se demostró que el implante elaborado sea por manufactura aditiva o por forja, fue compatible con las normas internacionales. Se realizaron 84 estudios de rayos $X$ en 18 cuellos de porcino. En el $100 \%$ de las columnas cervicales de los porcinos, el rango en flexión fue menor que el de extensión. Existió mayor rango de movimiento cuando se utilizaron dos implantes y cuando el implante fue más grande. Conclusiones: El implante cervical flexible elaborado por forja o por manufactura aditiva de titanio ELI presentó resultados prometedores, por lo que podria postularse su uso experimental en pacientes tributarios de artroplastía cervical.

Palabras clave: Prótesis e Implantes; Artroplastia de Reemplazo; Diseño de Prótesis; Ensayo de Materiales; Estructuras Animales; Modelos Animales (fuente: DeCS BIREME).

\begin{abstract}
Introduction: Cervical arthroplasty is a surgical procedure that involves the use of disc implants with the capacity of preserving segmental movement. It is a challenge for countries like ours to develop alternatives to the high costs of the aforementioned implants, with patented designs, feasible to manufacture, with biocompatibility, safety, and that simulates as much as possible the physiological cervical movements. Objectives: To develop the geometry and mechanics of a patented disc design elaborated with titanium ELI by additive manufacturing, as well as, to verify its compatibility with the physiology of cervical movements. Methods: The study design had three iterative stages: the first one was the development of the implant geometry and the finite-element study using international standards as a reference. In second place, studies of metallography, characterization of the material and fatigue tests of the implant were developed. Finally, a cadaverous animal model was used to confirm the compatibility of the implant with cervical movements. Results: The implants manufactured by forging or by additive manufacturing are compatible with international standards. 84 x-ray studies were performed on 18 pig necks. In $100 \%$ of the cervical columns of the pigs, the range in flexion was smaller than that of extension. There was a greater range of motion when two implants were used and when the implant was larger. Conclusions: The flexible cervical implant made by forging or a titanium-ELI additive manufacturing have promising results, because of that, an experimental use in patients, who needs cervical arthroplasty, could be considered.
\end{abstract}

Keywords: Prostheses and Implants; Arthroplasty, Replacement; Prosthesis Design; Materials Testing; Animal Structures; Models, Animal (source: MeSH NLM). 


\section{INTRODUCCIÓN}

La artroplastía vertebral cervical es una técnica operatoria neuroquirúrgica que tiene como objetivo preservar el movimiento entre dos vértebras cervicales contiguas, luego de la cirugía de resección discal total, en el tratamiento de la hernia del núcleo pulposo cervical o de la enfermedad degenerativa cervical ${ }^{(1)}$. Aunque el tema se encuentra en estudio continuo, se ha demostrado que la artroplastía es más útil que la artodesis para reducir el riesgo de enfermedad de los discos adyacentes ${ }^{(2)}$.

La artroplastía cervical implica el uso de un implante discal elaborado con material o materiales biocompatibles que tengan la característica de permitir el movimiento de las vértebras adyacentes, estables mecánicamente durante su uso, que no claudiquen en el tiempo (llegando a considerarse de uso perpetuo), que no permitan osificación heterotópica y que sean compatibles con su uso simultáneo en más de un nivel discal, sean del mismo tipo o en asociación con otros tipos de implantes ${ }^{(3,4)}$. Estos implantes discales pueden clasificarse de acuerdo a su configuración, así como de los materiales de los que están hechos. Adicionalmente, los diseños actuales se construyen alrededor de un cojinete. En general los implantes pueden ser: de una pieza, metal-metal o metal-polímero y también pueden clasificarse en cuatro tipos: móviles, inamovibles, restringidos o $1 \mathrm{P}$ (de una sola pieza) ${ }^{(2)}$. Los implantes inamovibles implican que sus partes no se mueven excepto el deslizamiento de la zona esférica en su soporte, pero los móviles y los restringidos permiten movimiento de la zona esférica ${ }^{(5)}$.

Nuestro grupo de investigación trabajó la optimización de la geometría tridimensional y realizó tanto el estudio de elementos finitos, así como las pruebas mecánicas de un implante diseñado y patentado como modelo de invención en el Perú (Título $N^{\circ} 0704$, clasificación A61F 2/44), con la finalidad de transformarse en una alternativa para el tratamiento de estos casos en la medida de lograr la creación de un dispositivo con más grados de movimien- to que los predecesores. De esta manera, el nuevo implante diseñado denominado implante cervical flexible ( $\mathrm{FCl}$ ) permitiría movimientos de flexión, extensión, bending lateral e incluso deformarse con cargas axiales. El diseño se basa en la modificación geométrica y mejora funcional de los implantes existentes en el mercado, de modo tal que se utilizó la aleación de titanio, aluminio y vanadio, denominandose a esta aleación: titanio ELI, con probada biocompatibilidad y compatibilidad con la resonancia magnética hasta ciertos niveles de uso común en las imágenes de columna vertebral ${ }^{(6)}$. Por ende, el $\mathrm{FCl}$ se basa en un diseño de una sola pieza que evita la posibilidad que sus componentes se separen durante el movimiento cotidiano de la columna vertebral o incluso por accidentes.

La justificación del diseño del $\mathrm{FCl}$ fue disponer de la opción de un implante de menor precio en el mercado peruano, ya que los implantes utilizados hasta la actualidad son manufacturados en el extranjero, tienen limitaciones funcionales y su comercialización representa costos elevados para el poder adquisitivo. Un estudio de mercado, realizado previamente, permitió estimar que en la actualidad se colocan 320 implantes discales cervicales mensuales en nuestro país, en las que, el 53\% de los neurocirujanos utilizan un implante 1P en sus cirugías; en contraste, el $73 \%$ de los que no usan este tipo de implantes lo harían si el precio fuera menor ${ }^{(7)}$.

El objetivo del estudio fue el desarrollo de la geometría y mecánica del diseño del implante cervical flexible así como verificar su compatibilidad con la fisiología de los movimientos cervicales en un modelo cadaverico animal.

\section{MÉTODOS}

Se realizó un estuido observacional descriptivo en tres etapas: la primera, el desarrollo de la geometría del implante y el estudio de elementos finitos utilizando como referencia normas internacionales; en segundo lugar, se desarrollaron estudios de metalografía, caracterización del material incluyendo pruebas de fatiga del implante; y finalmente, se utilizó un modelo animal cadavérico para confirmar la compatibilidad del implante con los movimientos cervicales

\section{Desarrollo del implante cervical flexible ( $\mathrm{FCl})$}

El FCl es una estructura metálica que se caracteriza por estar constituido por un monoblock de una lámina doblada sobre sí misma tomando la forma de S, que tiene cuatro caras disímiles entre sí, tal como se muestra en las figuras 1, 2 y 3 . Este implante está elaborado de una aleación de titanio, aluminio y vanadio (Ti6AL4V) grado ELI (extra low interstitial), que se considera como la aleación de mejor calidad para uso en humanos ${ }^{(8)}$. El $\mathrm{FCl}$ está manufacturado por impresión tridimensional o por forja en 12 tamaños: pequeño, mediano, grande y extragrande, de tres alturas cada uno de ellos: 5, 6 y $7 \mathrm{~mm}$. Tiene como objetivo ser utilizado de forma permanente como reemplazo del disco vertebral cervical por vía anterior cuando sea necesario ser resecado como consecuencia de haber sufrido proceso degenerativo discal, estenosis de canal cervical central, foraminal o degeneración traumática que haya ocasionado su herniación o deformidad con consecuente dolor cervical y braquial o incluso signos de compresión de la médula espinal cervical o la raíz nerviosa.

Adicionalmente, el $\mathrm{FCl}$ tiene la singularidad de permitir movimientos de la columna vertebral cervical de flexo-extensión anteroposterior, flexión lateral, y permitir la compresión en la sobrecarga axial vertebral. Debido a la morfología de las vértebras de la columna cervical este implante puede ser usado en desde el disco C3/C4 hasta el nivel C7/D1 inclusive. Tiene la característica de poder ser implantado a través de un abordaje cervical anterior convencional y es autosostenible en el espacio discal cervical debido que una vez puesto en él, bajo la compresión del porta-implante y la distracción de las vértebras contiguas, recupera su altura final y sus dientes transversales lo adhieren a los platillos discales adyacentes por lo que no requiere de tornillos $u$ otros implantes adicionales. 


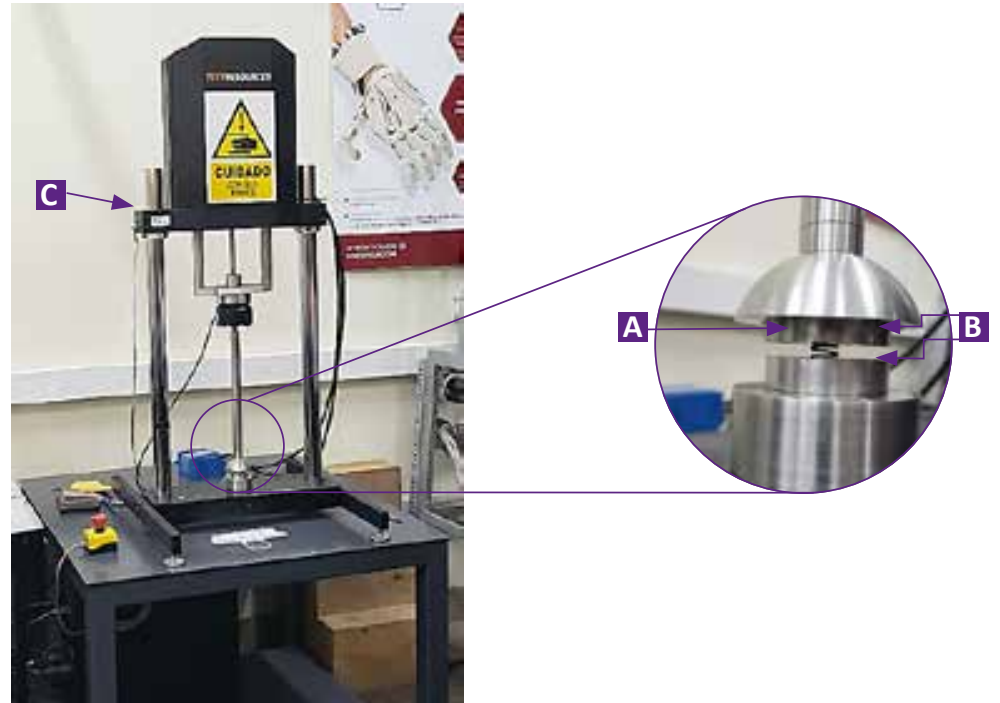

Figura 1. Implementación del equipo para el ensayo de flexión-compresión del prototipo. A. Prototipo del implante, B. Accesorios de sujeción y posicionamiento del implante, C. Actuador y sensor de máquina universal de tracción.

Mejoramiento de la estructura geométrica y análisis por elementos finitos

A partir del diseño de la patente se realizaron modificaciones de la geometría utilizando el software Autodesk Inventor $2019{ }^{\circledR}$ y también simulaciones computacionales usando el método de los elementos mediante el software ANSYS Inc
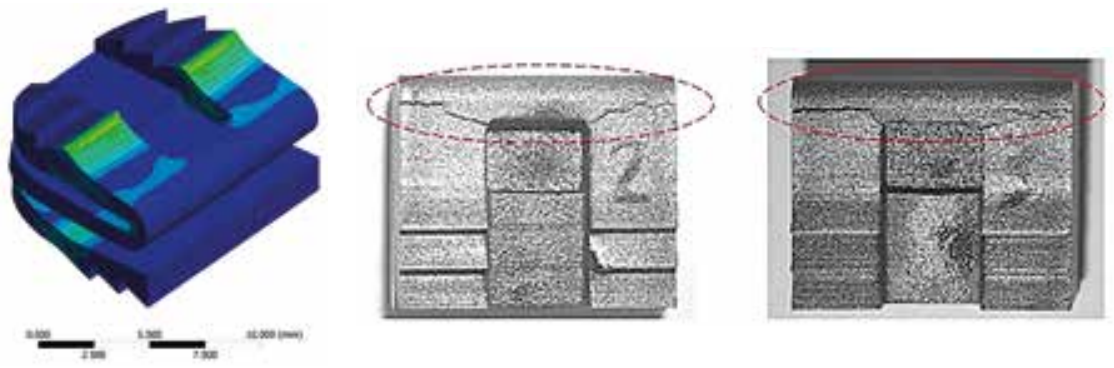

Figura 2. Modelo preliminar de elementos finitos (coloreado) y dos de los prototipos que se rompieron en las pruebas preliminares de fatiga.
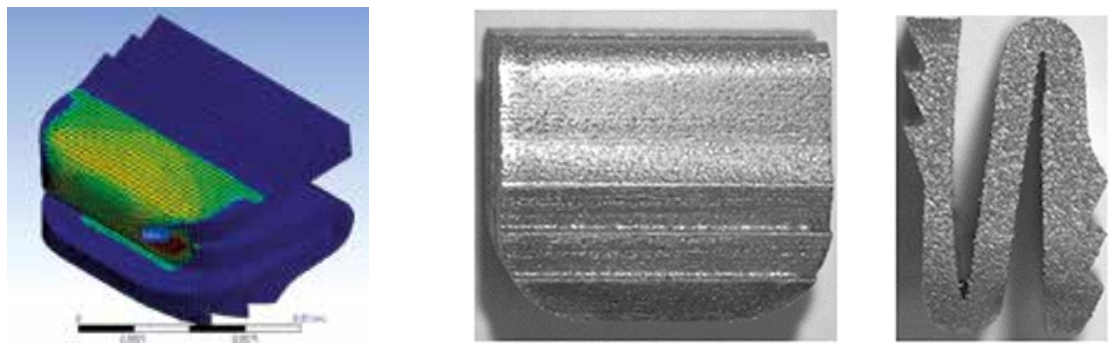

Figura 3. Modelo final de elementos finitos (coloreado) y el implante que toleró las pruebas dinámicas luego de suprimir la ranura hecha para el portaimplante.
Para poder realizar el cálculo mediante software de elementos finitos, primero se definió un modelo geométrico en 3D; y para la optimización de la geometría, se realizaron una serie de iteraciones con diferentes espesores y diferentes formas que variaban el diseño original, tanto en tamaño como en radios de curvatura, chaflanes, espesores del material. Se procedió a mitigar los concentradores de tensiones, se utilizaron redondeos en las zonas de mayor cambio de sección, y también se cambiaron los espesores del implante hasta obtener la combinación más adecuada entre carga y deformación. Adicionalmente, durante las iteraciones se desarrollaron modificaciones de la geometría del implante que derivaron en un modelo final en el cual se eliminaron las ranuras superior e inferior antes diseñadas para el porta-implante y en su reemplazo se crearon sendas lengüetas de apoyo que cumplían con la misma función.

\section{Estudio mecánico del implante}

El estudio mecánico se realizó en dos etapas: metalografía de la aleación y caracterización del material base. En la primera etapa, se estudió el titanio ELI mediante un microscopio óptico Leica DMI500M ${ }^{\circledR}$ equipado con una cámara digital LEICA ${ }^{\circledR}$. Las muestras fueron montadas en resina epóxica y sometidas a un procedimiento de desbaste, la reacción química empleada para revelar la microestructura fue coloreado con $\mathrm{NH} 4 \mathrm{HF} 2+\mathrm{H} 2 \mathrm{O}+$ etanol. Mientras que en la segunda etapa, se caracterizó el material base Ti6AI4V ELI, fabricado por tecnología aditiva. Esta tecnología provee a las probetas fabricadas, un acabado rugoso y una ligera capa de óxido que cubre a estas probetas. La mencionada caracterización se realizó mediante las siguientes pruebas:

- Análisis químico, mediante la espectrometría de emisión óptica por chispa (marca Bruker ${ }^{\circledR}$ modelo Magellan Q8) siguiendo los lineamientos de la ASTM E2994-16 (9).

Ensayo de tracción en dos grupos de probetas: uno que se ensayó tal como fueron obtenidas del proceso de fabricación; y el segundo grupo, con probetas previamente pulidas. 
Los ensayos se realizaron según los lineamientos que establece la norma ASTM E8 ${ }^{(10)}$, para cumplir los requerimientos de una aleación de Ti6Al4V ELI, con la ayuda de una máquina universal de ensayos (marca Zwick Roell ${ }^{\circledR}$ modelo Z250). Los parámetros de velocidad para ensayo empleados fueron: 0,015 mm/min, como velocidad de ensayo según la norma ASTM E8, hasta alcanzar el esfuerzo de fluencia; y 0,2 mm/min, como velocidad general de ensayo.

- Ensayo de doblado a las probetas, de acuerdo a los lineamientos de la norma ASTM E290-14, con una máquina universal de tracción Zwick Roell ${ }^{\circledR} \mathrm{Z}$ 250 de alta precisión ${ }^{(11)}$.

- Ensayo de dureza a las probetas impresas por manufactura aditiva. Se empleó el método de dureza Rockwell según los lineamientos de la norma ASTM E18-17 ${ }^{(12)}$, con el apoyo de una máquina de dureza INSTRON $2002 T^{\circledR}$.

- Ensayo de fatiga, mediante una máquina de tracción universal marca Test Resources ${ }^{\circledR}$ con capacidad de $1 \mathrm{kN}$ de carga cíclica máxima (figura 1). Estos ensayos se realizaron siguiendo básicamente el mismo procedimiento que los realizados al primer prototipo y segundo prototipo; en tres implantes identificados bajo los siguientes parámetros: frecuencia de ensayo $10 \mathrm{~Hz}$; precarga de ensayo-20 N; carga principal-80 N; número de ciclos de ensayo, diez millones de ciclos.

Estas pruebas se realizaron considerando los requerimientos de normas internacionales específicas, buscando que el implante alcance un máximo de ciclos de fatiga, aunque no fueron realizadas en un ambiente temperado a $37^{\circ} \mathrm{C}$ y en una inmersión en cloruro de sodio al 0,9\% como idealmente se prescribe ${ }^{(13,14)}$.

\section{Prueba del implante en columna ver- tebral de porcino}

Se utilizaron 18 cuellos de porcino, los cuales fueron disecados cuidadosamente retirando los tejidos blandos perivertebrales hasta dejar expuesta la columna vertebral desde $\mathrm{C} 2$ hasta $\mathrm{C7}$, cumpliendo las normas internacionales y principios éticos relacionados al uso de animales de experimentación. Posteriormente, con ayuda de una mesa de rayos $X$ se procedió a numerar los especímenes. Se procedió a realizar la disectomía cervical de uno o dos niveles para evaluar los grados de flexión y de extensión que se lograba al aplicar una fuerza externa y para el monitoreo de los implantes en flexión y extensión. Adicionalmente, se demostró la respuesta del implante en el doblado lateral la columna vertebral cervical.

Se utilizaron tres tipos de implantes: pequeño $(P)$, mediano $(M)$ y grande $(G)$, todos ellos con $5 \mathrm{~mm}$ de altura. Los implantes fueron elaborados por manufactura aditiva por la empresa Spartacus $3 D{ }^{\circledR}$. Los implantes fueron colocados en profundidad discal de manera similar a una cirugía convencional evitando contactar el borde posterior del platillo vertebral, situándolos a $2 \mathrm{~mm}$ aproximadamente por detrás del borde anterior del platillo vertebral, tratando que el punto medio del implante coincida con un hipotético centro de rotación instantánea de la vértebra superior al disco intervenido.

Se procedió a realizar 84 estudios de rayos $X$ en posición lateral, neutra, lateral-flexión-máxima y lateral-extensiónmáxima. Para lograr los movimientos pasivos de los especímenes se introdujo en el canal vertebral, tanto en el extremo proximal como distal, sendas palancas metálicas. Estos estudios, a su vez, se encontraron distribuidos de acuerdo al tamaño de implante probado, en 45 de ellas se evaluó un solo implante: en 15 se realizaron en implantes $P, 15$ en implantes $\mathrm{M}$ y 15 en implantes G. Por otro lado, se ejecutaron 39 radiografías utilizando una combinación de dos implantes: 15 probando la combinación de implantes $\mathrm{P}+\mathrm{M}, 15$ en la combinación de $\mathrm{M}+\mathrm{G}$, y 9 con la combinación $\mathrm{P}+\mathrm{G}$.

El rango de movimiento (RM) se calculó por la diferencia aritmética entre el promedio de las cinco mediciones hechas en cada espécimen, tanto en posición flexión máxima versus neutra, y extensión máxima versus neutra; definiéndose máxima como aquella obtenida con la aplicación de fuerza manual intensa sin llegar a destruir las estructuras osteoligamentarias de la columna vertebral o cuando se objetivaba la máxima flexión o extensión de los implantes. Se obtuvo de esta manera un valor en grados sexagesimales en flexión y extensión para diferentes combinaciones de implantes utilizados.

La iteración, previamente planificada, consistía en que luego de la prueba mecánica en las que los prototipos no lograran alcanzar los cinco millones de ciclos, se modificaba el diseño del implante hasta lograr alcanzar la meta; esta iteración tuvo que ser realizada en tres oportunidades. Se verificó que los modelos realizados tanto por forja como por manufactura aditiva cumplían de forma similar con las características mecánicas requeridas.

\section{RESULTADOS}

\section{Mejoramiento de la estructura geométrica y análisis por elementos finitos}

Se realizó la simulación para las diferentes presentaciones del implante, utilizándose las presentaciones de las tallas P, M, G. Se utilizaron datos estándar de las propiedades mecánicas, así como de la curva de fatiga del material y de las condiciones de frontera necesarias para aproximar el comportamiento del implante. El diseño del implante utilizado en la simulación numérica presentó buen comportamiento en la aplicación de cargas cíclicas en un análisis de fatiga.

\section{Estudio mecánico del implante}

Desde el análisis metalográfico descrito líneas arriba, se logró apreciar claramente que la microestructura se encuentra dispuesta por capas, siendo el espesor de cada capa de 30 micrómetros. También se demostró que a elevados aumentos (a partir de 500x) se distinguió la fase alfa acicular, en una matriz de fase beta, lo cual corresponde a una aleación Ti-6Al-4V ELI bifásica alfa y beta; la fase alfa presenta una red hexagonal con empaquetamiento compacto, mientras que la fase beta presenta una red cúbica centrada en el cuerpo.

Por otro lado, de acuerdo con los valores obtenidos del porcentaje en peso de los elementos que constituyen la mues- 
tra analizada, la composición química se encontró dentro de los parámetros que se especifican en la norma ASTMF136-13. Adicionalmente, se encontró que las probetas cumplen con los requerimientos de la norma AST- F136 en cuanto a la resistencia a la tracción. En el ensayo de doblado de las probetas impresas por manufactura aditiva se demostró que ninguna probeta presentó discontinuidades en la zona de mayor deformación, por lo que los resultados fueron conformes. En el ensayo de dureza de las probetas se demostró que el material corresponde a una aleación Ti6Al4V ELI para aplicaciones médicas según normas internacionales.

Con respecto al ensayo de fatiga, en las dos primeras iteraciones los resultados no fueron favorables y los prototipos fallaron (figura 2). En la tercera iteración, corrigiendo la geometría del implante al no incluir la ranura para el porta-implante en las caras superior e inferior del mismo, se logró que las muestras alcancen los diez millones de ciclos a una frecuencia de ensayo de $10 \mathrm{~Hz}$, precarga de ensayo de-20 N, y carga principal de-80N (Figura 3).

Del estudio del implante en el modelo cadaverico animal

Se objetivó en todas las combinaciones de implantes realizados (100\%), que el rango en flexión fue menor que el de extensión, probablemente relacionado a la propia morfología y fisiología de la columna vertebral cervical del cerdo como animal cuadrúpedo en el que la extensión cervical es menor en condiciones normales, que en la flexión (Tabla 1).

Se realizaron 39 radiografías utilizando una combinación de dos implantes: 15 probando la combinación de implan-

Tabla 1. Mediciones de los ángulos de los platillos vertebrales cervicales adyacentes, expresados en grados sexagesimales, de los tres tipos de movimientos logrados en las columnas vertebrales cervicales de porcinos con uno o dos implantes.

Tamaño RN

\begin{tabular}{|c|c|c|c|c|c|c|c|c|}
\hline & & 1 & 2 & 3 & 4 & 5 & & \\
\hline \multirow[t]{4}{*}{$P$} & $\mathrm{~F}$ & 170 & 179 & 179 & 178 & 180 & 177 & $9^{\circ}$ \\
\hline & $E$ & 160 & 110 & 158 & 165 & 148 & 148 & $21^{\circ}$ \\
\hline & $\mathrm{N}$ & 167 & 169 & 160 & 180 & 168 & 169 & \\
\hline & & 6 & 7 & 8 & 9 & 10 & & \\
\hline \multirow[t]{4}{*}{$M$} & $\mathrm{~F}$ & 185 & 185 & 182 & 180 & 180 & 182 & $3^{\circ}$ \\
\hline & $E$ & 150 & 180 & 180 & 154 & 170 & 167 & $13^{\circ}$ \\
\hline & $\mathrm{N}$ & 180 & 180 & 187 & 170 & 180 & 179 & \\
\hline & & 11 & 12 & 13 & 14 & 15 & & \\
\hline \multirow[t]{4}{*}{ G } & $\mathrm{F}$ & 185 & 180 & 180 & 180 & 180 & 181 & $1^{\circ}$ \\
\hline & $E$ & 173 & 151 & 160 & 170 & 170 & 165 & $15^{\circ}$ \\
\hline & $\mathrm{N}$ & 180 & 180 & 180 & 180 & 180 & 180 & \\
\hline & & 16 & 17 & 18 & & & & \\
\hline \multirow[t]{4}{*}{$P+G$} & $\mathrm{~F}$ & 162 & 185 & 180 & - & - & 176 & $12^{\circ}$ \\
\hline & $\mathrm{E}$ & 140 & 150 & 140 & - & - & 143 & $20^{\circ}$ \\
\hline & $\mathrm{N}$ & 152 & 180 & 159 & - & - & 164 & \\
\hline & & 1 & 2 & 3 & 4 & 5 & & \\
\hline \multirow[t]{4}{*}{$\mathrm{P}+\mathrm{M}$} & $\mathrm{F}$ & 178 & 179 & 179 & 178 & 180 & 179 & $23^{\circ}$ \\
\hline & $E$ & 151 & 135 & 140 & 143 & 122 & 138 & $18^{\circ}$ \\
\hline & $\mathrm{N}$ & 152 & 155 & 152 & 152 & 168 & 156 & \\
\hline & & 6 & 7 & 8 & 9 & 10 & & \\
\hline \multirow[t]{3}{*}{$M+G$} & $\mathrm{~F}$ & 1192 & 195 & 195 & 198 & 194 & 195 & $23^{\circ}$ \\
\hline & $E$ & 140 & 175 & 175 & 133 & 151 & 155 & $17^{\circ}$ \\
\hline & $\mathrm{N}$ & 180 & 180 & 175 & 145 & 180 & 172 & \\
\hline
\end{tabular}

P: pequeño, M: mediano, G: grande; F: flexión, E: extensión, N: neutral. RM: rango de movimiento definido como la diferencia aritmética entre el promedio de las cinco mediciones hechas en cada espécimen, tanto en posición flexión máxima versus neutra, y extensión máxima versus neutra. Los números del 1 al 18 representan los especímenes cervicales porcinos estudiados.

tes $\mathrm{P}+\mathrm{M}, 15$ en la combinación de $\mathrm{M}+\mathrm{G}$, y 9 con la combinación $P+G$. Todas ellas tuvieron mayor RM respecto a donde se usó sólo un implante: $12^{\circ}, 23^{\circ}, 26^{\circ}$ versus $9^{\circ}, 3^{\circ}, 1^{\circ}$, no observándose mayor diferencia en el movimiento en extensión, lo cual se puede explicar tanto a una consecutiva resección del ligamento longitudinal anterior, así como a la sumatoria de efecto de la suma de la extensión de ambos implantes. También se registró mayor RM en la medida que el implante fue más grande $\left(9^{\circ}>3^{\circ}>1^{\circ}\right)$. Se demostró adicionalmente, que los implantes lograron un RM de flexión antero-posterior en $90^{\circ}$, extensión antero-posterior $70^{\circ}$, flexión lateral en un rango de $20^{\circ}$ a $45^{\circ}$ y hasta $90^{\circ}$ de rotación a cada lado (Figura 4).

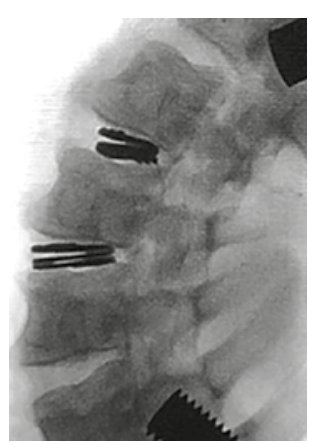

(a)

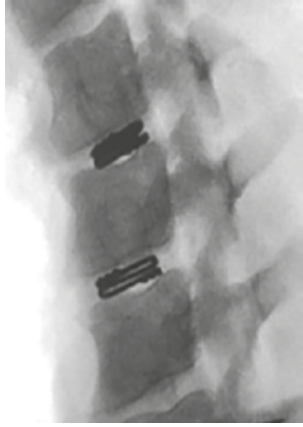

(b)

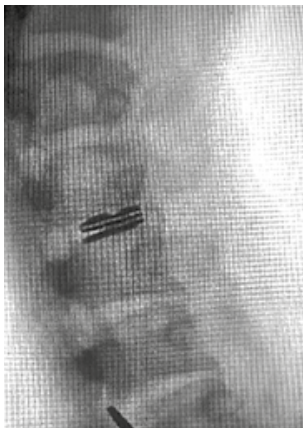

(c)

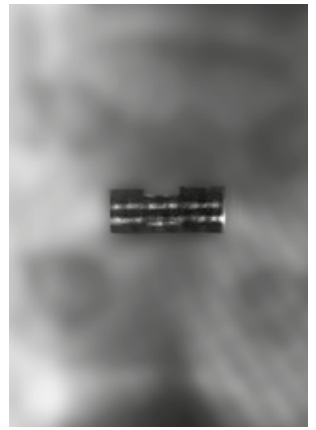

(d)

Figura 4. Rayos X del implante en el modelo animal: en (a) extensión antero-posterior, (b) flexión antero-posterior, (c) en flexión lateral, (d) vista antero-posterior. 

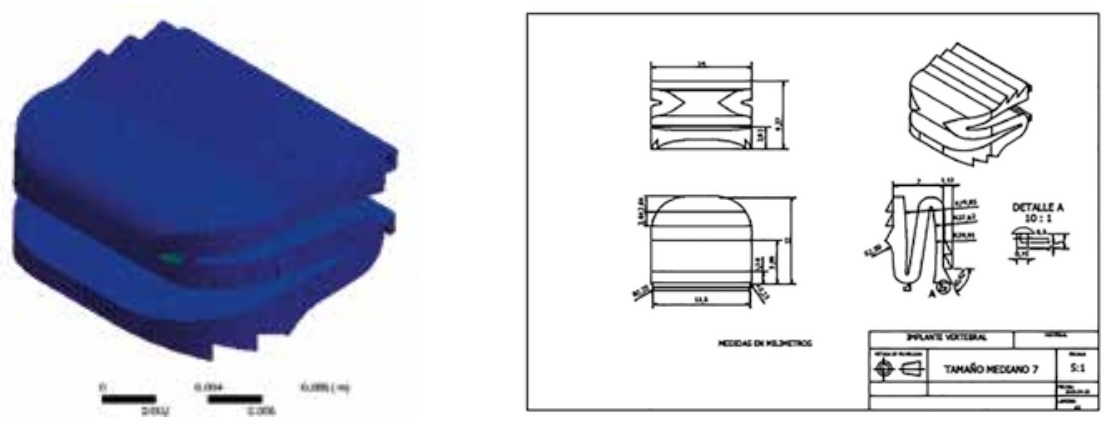

Figura 5. Modelo final de elementos finitos con lengüetas de apoyo para el porta-implante.

En ningún caso se produjo ruptura de los implantes o se ocasionó daño de los platillos vertebrales, estructuras articulares o ligamentarias de la columna vertebral. Durante el trabajo de colocar adecuadamente los implantes se evidenció que el máximo funcionamiento de los implantes ocurrió cuando se utilizó en un nivel o en dos, colocandolos por detrás de su supuesto eje de rotación.

\section{DISCUSIÓN}

Está demostrado el beneficio de la artroplastía, aunque en la actualidad el criterio es que podría incluso combinarse con la artrodesis para lograr buenos resultados en los pacientes ${ }^{(15)}$. Sin embargo, esto no soluciona problemas como la hipermotilidad generada por los implantes dinámicos clásicos en el cual una superficie pivotea sobre la otra ${ }^{(16)}$. Nuestro implante tiene la ventaja que al ser de una sola pieza, no es posible que se desensamble en situaciones de estrés fisiológico o por traumatismos y se ha demostrado que puede tolerar diez millones de ciclos de compresión sin romperse.

Adicionalmente, se debe considerar que la artroplastía cervical tiene por objetivo preservar el movimiento del segmento discal intervenido, por lo que se han desarrollado varios implantes denominados dinámicos, que se basan en dos o más partes móviles que pivotean entre sí y que incluso logran desplazarse en sentido antero-posterior para simular al máximo la dinámica fisiológica de la columna vertebral cervical e incluso su envejecimiento ${ }^{(17,18)}$. Aunque aún existe discusión respecto a los beneficios obte- nidos por el tratamiento con esta técnica para dos o tres espacios discales simultáneamente ${ }^{(15,19)}$. Se sabe incluso que no es la técnica más utilizada por grupos de neurocirujanos en el mundo, principalmente debido a los elevados costos, aunque se tiene proyección que esta situación se revertirá en los próximos años, ante la evidencia de los buenos resultados en el corto, mediano y largo plazo ${ }^{(20,21)}$. Por otro lado, la motilidad segmentaria o el alineamiento cervical general es afectado por la artroplastía de un nivel, por ende, los implantes discales cervicales deben tener la característica de simular la fisiología del movimiento cervical, lo que justifica la realización de pruebas de movimientos de flexión antero-posterior, extensión antero-posterior, flexión lateral, así como de tolerancia a la sobrecarga axial ${ }^{(22,23)}$. Por ello, los resultados obtenidos por el $\mathrm{FCl}$, en las pruebas realizadas en columna cervical de porcino, revisten especial importancia ya que corroboran la naturaleza flexible del implante probado.

Sólo existe en el mercado un implante hecho de una sola pieza, con forma de letra $C$ que sería mejor definido como un implante flexible o quizás como un intermedio entre la artrodesis cervical anterior y el reemplazo discal cervical ${ }^{(24)}$. Desde el punto de vista biomecánico, si bien muestra tendencia a estabilizar el segmento en flexión-extensión antero-posterior permitiendo movilidad residual, en la flexión lateral reduce significativamente el movimiento, aunque está establecido que a largo plazo tiene un excelente resultado con restauración de la calidad de vida del paciente ${ }^{(24,25)}$. Por otro lado, la manufactura aditiva por láser, también cono- cida como LAM, por sus siglas en inglés, es una relativamente novedosa técnica para elaborar piezas metálicas de uso en cirugía y teniendo en cuenta los detalles específicos en su tratamiento, puede ser perfectamente utilizada para la creación de implantes discales ${ }^{(26,27)}$. Adicionalmente, la adición de aleación de titanio (ELI) ha demostrado ser el tipo que posee mayor límite de fatiga, siendo un material bioinerte, con excelente resistencia a la corrosión, que puesto en una superficie ósea crea una intercara cerámica bioinerte que permite un excelente anclaje al tejido óseo vecino ${ }^{(28)}$.

Existen en la actualidad cinco tipos de aleaciones de titanio de uso médico que varían entre sí por su contenido de impurezas como el nitrógeno, carbono, hidrógeno, hierro y oxígeno. El aluminio le brinda a la aleación la tendencia a estabilizar la fase alfa, mientras que el vanadio estabiliza la fase beta como se evidenció en nuestro estudio metalográfico; además, la delgada capa de óxido, compacta y adherente, estabiliza el material y bajo condiciones fisiológicas determina una velocidad de corrosión muy baja (28).

Uno de los aspectos más novedosos de este estudio ha sido el planteamiento de una forma iterativa para corregir el diseño original del implante, antes de pasar a la manufactura de los primeros prototipos. Los resultados permitieron definir el grosor de la aleación del implante y la propia geometría, estableciéndose que debía eliminarse la ranura diseñada para el porta-implante, y creando sendas lengüetas de apoyo en la cara anterior para poder sostener y comprimir el implante en el momento de su colocación en el espacio discal (Figura 5).

Adicionalmente, parte importante del estudio tuvo como objetivo demostrar la viabilidad preliminar de la geometría del implante. La prueba de elementos finitos permitió un acercamiento bastante aproximado a la realidad de las pruebas de fatiga y logró corregir los modelos geométricos previos. En la actualidad incluso se logra con este método estimar las fuerzas musculares y las cargas internas para evaluaciones más precisas ${ }^{(29)}$, considerando 
que las cargas compresivas incrementan durante la flexión y extensión, deportes de contacto y otras actividades de la vida diaria, estableciéndose que van desde $120 \mathrm{~N}$ hasta $1200 \mathrm{~N}^{(13)}$.

Adicionalmente el implante debería tener un perfil sobre el borde anterior de las vértebras adyacentes al espacio discal intervenido de por lo menos $2 \mathrm{~mm}$, lo cual se logra sin inconvenientes por nuestro implante. Tanto los prototipos manufacturados por forja, por impresión láser 3D o manufactura, cumplieron con lo requerido por los estándares internacionales, debiendo mencionar que los implantes hechos por manufactura aditiva se sometieron a un tratamiento térmico denominado Isostatic Hot Pressing, el cual reduce la ansiotropía, optimizando las propiedades mecánicas, sobre todo la resistencia a la fatiga ${ }^{(26)}$. Nuestro implante, al tener forma de $S$ permite tanto el movimiento en la carga axial, flexión antro-posterior, extensión antero-posterior, así como en la flexión lateral como se ha demostrado en las pruebas de fatiga y en las pruebas en columna cervical de porcino.

Para definir con precisión las características clínicas de la aplicación del referido implante; asimismo, continua siendo un desafío el desarrollo de patentes de nuevos implantes cervicales en el mercado peruano y mundial.

Concluimos que el implante discal flexible, presentado en el presente artículo, es potencialmente útil para la artroplastía cervical, siendo elaborado de una sola pieza y de titanio ELI, sea por forja o por manufactura aditiva y tolera la fatiga durante su uso ad-libitum, pudiendo utilizarse hasta dos de ellos simultáneamente sin ocasionar daño en los platillos vertebrales o en las demás estructuras articulares y ligamentarias.

\section{AGRADECIMIENTOS}

Al ingeniero Juan Pedro Reyes Soto de la Pontifica Universidad Católica del Perú, por su colaboración en la formulación del proyecto. A la magíster Norma Salas Mejla por su colaboración en la corrección del presente manuscrito.

\section{REFERENCIAS BIBLIOGRÁFICAS}

1. Baaj AA, Uribe JS, Vale FL, Preul MC, Crawford NR. History of cervical disc arthroplasty. Neurosurg Focus. 2009;27(3):E10. DOI: 10.3171/2009.6.FOCUS09128

2. Nunley PD, Coric D, Frank KA, Stone MB. Cervical Disc Arthroplasty: Current Evidence and Real-World Application. Neurosurgery. 2018;83(6):1087-106. DOI: 10.1093/neuros/nyx579

3. Traynelis VC. Spinal arthroplasty. Neurosurgical Focus. 2002;13(2):1-7. DOI: 10.3171/foc.2002.13.2.11

4. American Academy of Orthopaedic Surgeons. Cervical Disc Arthroplasty. A technology Overview. [On line]. AAOS; 2010

5. Reeks J, Liang $\mathrm{H}$. Materials and Their Failure Mechanisms in Total Disc Replacement. Lubricants. 2015;3(2):346-64. DOI: 10.3390/lubricants3020346

6. EOS GmbH - Electro Optical Systems. Material Data Sheet. EOS Titanium Ti64ELI. [On line]. EOS; 2014 [citado 26 agosto 2019].

7. Knower IO. Informe del Estudio de Mercado del Prototipo de Implante Cervical Flexible (FCI) para e Instituto del Cerebro y la Columna Vertebral. 2019

8. Smith HE, Wimberley DW, Vaccaro AR. Cervical arthroplasty: material properties. Neurosurgical Focus. 2004;17(3):1-21. DOI: 10.3171/foc.2004.17.3.3

9. E01 Committee. Standard Test Method for Analysis of Titanium and Titanium Alloys by Spark Atomic Emission Spectrometry and Glow Discharge Atomic Emission Spectrometry (Performance-Based Method). West Conshohocken, PA: ASTM International; 2016 [citado 15 agosto 2019]. Disponible: http:// www.astm.org/cgi-bin/resolver.cgi?E2994-16

10. E28 Committee. ASTM E8 / E8M-16a, Test Methods for Tension Testing of Metallic Materials. West Conshohocken, PA: ASTM International; 2016 [citado 15 agosto 2019]. Disponible: http://www.astm.org/ cgi-bin/resolver.cgi?E8E8M-16A

11. E28 Committee. ASTM E290-14,Test Methods for Bend Testing of Material for Ductility. West Conshohocken, PA: ASTM International; 2014 [citado 15 agosto 2019]. Disponible: http://www.astm.org/ cgi-bin/resolver.cgi?E290-14

12. E28 Committee. ASTM E18-17, Test Methods for Rockwell Hardness of Metallic Materials. West Conshohocken, PA: ASTM International; 2017 [citado 15 agosto 2019]. Disponible: http://www.astm.org/ cgi-bin/resolver.cgi?E18-17

13. F04 Committee. ASTM F2346-18, Test Methods for Static and Dynamic Characterization of Spinal Artificial Discs. West Conshohocken, PA: ASTM International; 2018 [citado 15 agosto 2019]. Disponible: http://www.astm.org/cgi-bin/resolver.cgi?F2346-18

14. F04 Committee. ASTM F2423-11(2016), Guide for Functional, Kinematic, and Wear Assessment of Total Disc Prostheses. West Conshohocken, PA ASTM International; 2016 [citado 15 agosto 2019]. Disponible: http://www.astm.org/cgi-bin/resolver. cgi?F2423-11R16

15. Laratta JL, Shillingford JN, Saifi C, Riew KD. Cervical Disc Arthroplasty: A Comprehensive Review of Single-Level, Multilevel, and Hybrid Procedures. Global Spine J. 2018;8(1):78-83. DOI: 10.1177/2192568217701095

16. Ferrara LA. Advances in Orthopedics [On line]. 2012. The Biomechanics of Cervical Spondylosis [citado 15 agosto 2019]. Disponible: https://www. hindawi.com/journals/aorth/2012/493605/
17. Jaramillo-de la Torre JJ, Grauer JN, Yue JJ. Update on cervical disc arthroplasty: where are we and where are we going? Curr Rev Musculoskelet Med. 2008;1(2):124-30. DOI: 10.1007/s12178-008-9019-2

18. Richards O, Choi D, Timothy J. Cervical arthroplasty: the beginning, the middle, the end? British Journal of Neurosurgery. 2012;26(1):2-6. DOI: 10.3109/02688697.2011.595846

19. Chin-See-Chong TC, Gadjradj PS, Boelen RJ, Harhangi BS. Current practice of cervical disc arthroplasty: a survey among 383 AOSpine International members. Neurosurg Focus. 2017;42(2):E8. DOI: 10.3171/2016.11.FOCUS16338

20. Lu Y, McAnany SJ, Hecht AC, Cho SK, Qureshi SA. Utilization trends of cervical artificial disc replacement after FDA approval compared with anterior cervical fusion: adoption of new technology. Spine. 2014;39(3):249-55. DOI: 10.1097/ BRS.0000000000000113

21. Chang H-K, Chang C-C, Tu T-H, Wu J-C, Huang W-C, Fay L-Y, et al. Can segmental mobility be increased by cervical arthroplasty? Neurosurgical Focus. 2017;42(2):E3. DOI: 10.3171/2016.10. FOCUS16411

22. Tan LA, Riew KD, Traynelis VC. Cervical Spine Deformity-Part 1: Biomechanics, Radiographic Parameters, and Classification. Neurosurgery. 2017;81(2):197-203. DOI: 10.1093/neuros/nyx249

23. Ibrahim E. The Effects of Neck Posture and Head Load on the Cervical Spine and Upper Extremities [Tesis]. 2015 [citado 15 agosto 2019]. Disponible: https://macsphere.mcmaster.ca/ handle/11375/18088

24. Shichang L, Yueming S, Limin L, Lei W, Zhongjie Z, Chunguang $Z$, et al. Clinical and radiologic comparison of dynamic cervical implant arthroplasty and cervical total disc replacement for single-level cervical degenerative disc disease. J Clin Neurosci. 2016;27:102-9. DOI: 10.1016/j.jocn.2015.05.072

25. Welke B, Hurschler C, Packheiser A, Richter B, Daentzer D. Biomechanical Concept of a Novel Dynamic Cervical Implant: First Biomechanical Comparison among Fusion, TDR and Dynamic Stabilization. [On line]. Bremen; 2010.

26. Wycisk E, Siddique S, Herzog D, Walther F, Emmelmann C. Fatigue Performance of Laser Additive Manufactured Ti-6Al-4V in Very High Cycle Fatigue Regime up to 109 Cycles. Front Mater. 2015;2. DOI: 10.3389/fmats.2015.00072

27. Qian M, Xu W, Brandt M, Tang HP. Additive manufacturing and postprocessing of Ti-6Al-4V for superior mechanical properties. MRS Bulletin. 2016;41(10):775-84. DOI: 10.1557/mrs.2016.215

28. Turel MK, Kerolus MG, Adogwa O, Traynelis VC. Cervical arthroplasty: what does the labeling say? Neurosurgical Focus. 2017;42(2):E2. DOI: 10.3171/2016.11.FOCUS16414

29. Li Y, Zhang Z, Liao Z, Mo Z, Liu W. Finite Element Analysis of Influence of Axial Position of Center of Rotation of a Cervical Total Disc Replacement on Biomechanical Parameters: Simulated 2-Level Replacement Based on a Validated Model. World Neurosurg. 2017;106:932-8. DOI: 10.1016/j. wneu.2017.07.079 